\title{
Efeito da Seleção Errônea de Machos sobre Ganhos Genéticos, Utilizando-se Simulação ${ }^{1}$ \\ Paulo Luiz Souza Carneiro ${ }^{2}$, Ricardo Frederico Euclydes ${ }^{3}$, Martinho de Almeida e Silva4,5, Paulo Sávio Lopes ${ }^{3,5}$, Robledo de Almeida Torres ${ }^{3}$, Antônio Policarpo Souza Carneiro², Rodolpho de Almeida Torres Filho 6
}

\begin{abstract}
RESUMO - Populações simuladas foram usadas para verificar o efeito de erros na seleção de machos sobre estimativas de ganhos genéticos, ao longo de dez gerações de seleção, usando o BLUP. Cinco níveis de erros na seleção de machos $(0,5,10,15$ e $20 \%)$ e três herdabilidades $(0,10 ; 0,30$; e 0,60$)$ foram trabalhados. Vinte machos e 200 fêmeas foram selecionados pelo BLUP. Após a seleção dos 20 machos em cada geração, 1, 2, 3 e 4 reprodutores foram substituídos por outros aleatoriamente. Os valores fenotípicos e a eficiência do BLUP com erro, em relação ao BLUP sem erro, foram reduzidos com o aumento no nível de erro. A redução dos ganhos genéticos, em função da inclusão deste tipo de erro, não apresentou, aparentemente, associação com a herdabilidade da característica.
\end{abstract}

Palavras-chave: BLUP, ganho genético, seleção, simulação

\section{Effect of Errors in the Selection of Males on the Genetic Gain Using Simulation}

\begin{abstract}
Simulated populations were used to verify the effect of errors in the selection of males in the estimates of genetic gains along ten generations of selection, using BLUP. Five levels of errors in the selection of males $(0,5,10,15$ and $20 \%)$ and three heritabilites $(.10, .30 \mathrm{e} .60)$ were used. Twenty males and 200 females were selected by BLUP. After the selection of 20 males in each generation, 1, 2, 3 and 4 sires were changed by others randomly. The phenotypic values and efficiency of BLUP with errors in relation to the BLUP without errors reduced with the increase in the error level. The decrease of the genetic gains in function of the inclusion of this type of error did not, apparently, present a relationship association with heritability of the trait.
\end{abstract}

Key Words: BLUP, genetic gain, selection, simulation

\section{Introdução}

A seleção é feita com base nos valores fenotípicos dos indivíduos e de seus parentes, que são coletados no "campo", geralmente provenientes de programas de melhoramento. Assim, o progresso genético pode ser influenciado não apenas por erros nos valores fenotípicos dos indivíduos e de seus parentes, como também por qualquer outro fator que acarrete redução de precisão na obtenção das informações utilizadas como critério de seleção. Como conseqüência imediata, têm-se a seleção de animais que não deveriam ser selecionados e a exclusão de animais que deveriam ser selecionados. No entanto, apesar de os trabalhos encontrados em literatura discutirem como o progresso genético obtido pela seleção por meio do BLUP pode ser influenciado por erros nos valores fenotípicos e erros de pedigree, nenhum trabalho mostra o efeito de determinado nível de erros na seleção de machos sobre os ganhos genéticos, tornando este estudo importante.

VAN VLECK (1970a e 1970b) mostrou que a identificação incorreta de progenitores, em dados de bovinos, pode provocar estimativas viesadas de herdabilidades e erros de avaliação de progenitores e de estimativa de progresso genético, durante o processo de seleção.

HENDERSON (1983) cita que a precisão no processo de seleção é de grande importância, especialmente nos casos em que os animais selecionados são usados intensivamente, como é o caso de touros em programas de inseminação artificial, em que pequeno número de indivíduos pode ter grande influência sobre o progresso genético.

LONG et al. (1990) usaram o coeficiente de correlação de Spearman para avaliar a seleção indi-

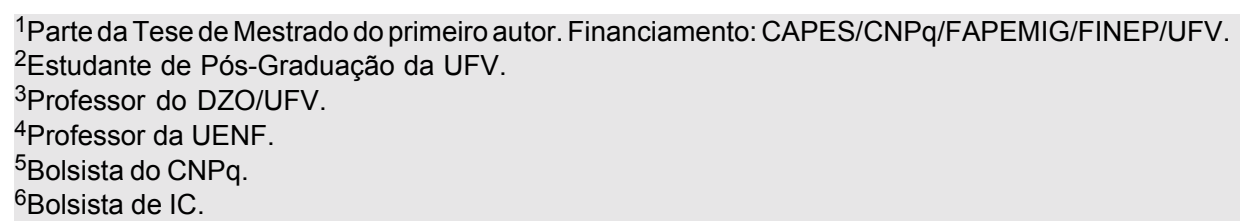


vidual, o índice de seleção e o BLUP, na presença de diferentes níveis de erros de pedigree, para características com herdabilidades 0,$13 ; 0,18$; e 0,53 . Esses autores verificaram maior diferença entre o ranking dos animais para a característica com herdabilidade igual a 0,13 , em que a correlação com o BLUP correto foi de 0,78 e 0,85 para seleção individual e índice de seleção, respectivamente. Entre os três métodos, o BLUP foi o mais influenciado pelos erros de pedigree. O coeficiente de correlação reduziu de 1,0 para 0,85 , quando o nível de erro passou de 0 para $20 \%$.

LIMA (1998) usou dados simulados para verificar os efeitos de erros nos valores fenotípicos sobre ganhos genéticos preditos e seus reflexos sobre a seleção dos indivíduos. O efeito de erros na seleção foi verificado comparando-se as ordens de classificação dos indivíduos, com e sem erro nos valores fenotípicos, além de observar o percentual de indivíduos que deixaram de ser selecionados quando os valores fenotípicos apresentavam erros. Observou, também, que, à medida que se aumentaram os níveis de erro, deixou-se de selecionar número crescente de melhores indivíduos.

O objetivo deste trabalho foi verificar o impacto da seleção errônea de machos sobre os ganhos genéticos ao longo de dez gerações de seleção, comparando o BLUP na presença de erro com o BLUP sem erro, utilizando populações simuladas.

\section{Material e Métodos}

Com o uso do programa GENESYS, desenvolvido por EUCLYDES (1996), foi simulado um genoma constituído de uma única característica quantitativa governada por 500 loci. O genoma simulado para o presente estudo apresentou os seguintes parâmetros: quinze pares de cromossomos de tamanhos aleatórios; três mil centimorgans de comprimento; os efeitos aditivos dos locos quantitativos foram simulados, segundo a distribuição normal; os locos quantitativos foram dialélicos e não possuíam desvios de dominância nem interações entre si; não possuíam cromossoma sexual e as freqüências gênicas iniciais eram iguais em ambos os sexos; a distribuição matemática usada para a simulação das freqüências iniciais dos locos quantitativos nas populações foi a uniforme, com média de 0,50 ; os efeitos fixos foram sexo, EF1 e EF2, sendo os dois últimos possuidores de 5 e 4 níveis ou classes, respectivamente; e os efeitos de ambiente não controláveis foram simulados conforme a distribuição normal.

A partir deste genoma foram simuladas três popu- lações bases, nas quais a característica quantitativa possuía herdabilidade inicial de 0,$10 ; 0,30$; e 0,60 , respectivamente. Foram simuladas populações bases constituídas de 800 animais (400 machos e 400 fêmeas).

Para construção das populações iniciais partiu-se das três populações bases, correspondente às herdabilidades estudadas, e escolharam-se, de forma aleatória, 20 machos e 200 fêmeas, que foram utilizados como progenitores das gerações seguintes. Para cada fêmea foram simulados oito descendentes, resultando em populações de 1.600 animais. Estas populações foram utilizadas para incorporação dos erros na seleção de machos.

Foram comparados os níveis de 0, 5, 10, 15 e 20\% de erros (substituição de machos selecionados por não-selecionados). Para simular este tipo de erro, implementou-se ao GENESYS um programa capaz de simular tal erro. A cada geração foram selecionados, utilizando-se o BLUP, 20 machos e 200 fêmeas. Após a seleção dos 20 machos, 1, 2, 3 e 4 reprodutores foram substituídos por outros, tomados de forma aleatória, entre os 780 machos não-selecionados da mesma geração, correspondendo a 5, 10, 15 e 20\% de erros, respectivamente. Este processo foi conduzido durante 10 gerações consecutivas e por 100 ciclos, ou repetições, para as três herdabilidades estudadas, sendo os diferentes níveis de erros incorporados às populações a cada geração.

Para escolha dos melhores animais, baseada no BLUP, foi utilizada a metodologia de modelos mistos proposta por HENDERSON (1963, 1973 e 1974), conforme apresentada por MARTINS et al. (1997). Os dados foram analisados utilizando-se o seguinte modelo estatístico:

$$
y_{i j k l}=\mu+A_{i}+B_{j}+S_{k}+g_{l(j i k)}+e_{l(j i k)}
$$

em que $\boldsymbol{y}_{i j k l}=$ observação do animal 1 , do sexo $\mathrm{k}$ referentes aos efeitos fixos EF1 i e EF2 j; $\mu=$ constante associada à todas observações; $A_{i}=$ efeito fixo do EF1, com i=1, 2, 3, 4, 5; $B_{j}=$ efeitos fixo do $E F 2$, $\operatorname{com} \mathrm{j}=1,2,3$, 4; efeito fixo do sexo $\mathrm{k}$, com $\mathrm{k}=1,2 ; \boldsymbol{g}_{1(i j k)}=$ efeito aleatório do indivíduo 1, no EF1 i, EF2 j e sexo k; e $\boldsymbol{e}_{1(i j k)}=$ erro aleatório associado a cada observação.

$\mathrm{Na}$ escolha dos animais para reprodução (20 machos e 200 fêmeas), selecionaram-se os animais que obtiveram maior $\hat{g}$, que representa o valor genético predito do indivíduo.

A eficiência do BLUP com erro em relação ao BLUP sem erro na seleção de machos foi avaliada utilizando-se a seguinte expressão: 


$$
E_{B B t}=\frac{\Delta G_{B t}}{\Delta G_{B(0) t}}
$$

em que $E_{B B t}$ representa a eficiência do BLUP com diferentes níveis de erros em relação ao BLUP com $0 \%$ de erro, na geração t; $\Delta G_{B t}$ é o ganho genético obtido pelo BLUP na geração $t$, para os diferentes níveis de erros; e $\Delta G_{B(0) t}$ é o ganho genético obtido pelo BLUP com $0 \%$ de erro, na geração t.

\section{Resultados e Discussão}

Os resultados de valores fenotípicos e de eficiência observados para as populações submetidas à seleção pelo BLUP com 0, 5, 10, 15 e 20\% de erro na seleção de machos, para as herdabilidades 0,$10 ; 0,30$; e 0,60 , por geração, são apresentados nas Tabelas 1 e 2 , respectivamente. Observa-se que os maiores valores fenotípicos e de eficiência foram obtidos nas populações submetidas ao BLUP sem erros, diminuindo à medida que se aumentou o erro na seleção dos machos.

Para característica com herdabilidade 0,10 , verificou-se redução média em ganho genético por geração de 1,9 a 8,4\% para o BLUP com erro, em relação ao BLUP sem erro. Esta redução, para a característica com herdabilidade 0,30 , variou de 1,7 a $7,5 \%$ e para característica com herdabilidade, 0,60 de 2,5 a 9,8\% (Tabela 3).

Como pode ser observado, a redução dos ganhos genéticos, em função da inclusão deste tipo de erro, não apresenta, aparentemente, associação com a herdabilidade da característica, como ocorre para erros de pedigree, fato também observado por CARNEIRO (1998). Isto é explicável, já que neste estudo a redução observada nos ganhos genéticos é conseqüência da substituição de machos selecionados por não-selecionados, não provocando, portanto, alterações nas informações de parentes. Assim, a intensidade com que os ganhos genéticos foram influenciados dependeu da qualidade do animal selecionado erroneamente (animal não-selecionado e incluído entre os selecionados para gerar o erro) e do desempenho do animal que deixou de ser selecionado (animal selecionado que foi substituído por animal não selecionado).

Apesar de não terem sido encontradas diferenças marcantes entre as reduções nos ganhos genéticos (máximo de 2,3\%), quando se compararam as diferentes herdabilidades, nas populações com erro na seleção de machos, deve-se lembrar que para características de baixa herdabilidade, em que as informações de parentes são mais importantes, há maior chance de se selecionarem animais erroneamente, graças à presença de erros de pedigree. LONG et al. (1990) mostraram, por meio da correlação de Spearman, que é possível obter diferenças na ordenação (ranking) dos selecionados para reprodução de $15 ; 12$, 9; e 5\%, quando se compara a ordenação dos selecionados a partir de dados sem erros de pedigree com dados contendo $20,15,10$ e $5 \%$ dos registros incorretos, respectivamente, para uma característica com herdabilidade 0,18 .

\begin{tabular}{|c|c|c|c|c|c|c|c|c|c|c|}
\hline \multirow{2}{*}{\multicolumn{2}{|c|}{$\begin{array}{l}\text { Método de seleção } \\
\text { Selection method }\end{array}$}} & \multicolumn{9}{|c|}{ Geração } \\
\hline & & \multicolumn{9}{|c|}{ Generation } \\
\hline & $\overline{1}$ & 2 & 3 & 4 & 5 & 6 & 7 & 8 & 9 & 10 \\
\hline & & \multicolumn{9}{|c|}{ Herdabilidade $($ Heritability $)=0,10$} \\
\hline $\operatorname{BLUP}(0 \%)^{1}$ & $-0,5$ & 6,5 & 15,8 & 24,7 & 33,3 & 41,6 & 49,5 & 57,4 & 64,8 & 71,7 \\
\hline $\operatorname{BLUP}(5 \%)^{1}$ & $-0,9$ & 6,0 & 14,7 & 23,9 & 32,6 & 40,8 & 48,8 & 56,5 & 64,3 & 71,7 \\
\hline $\operatorname{BLUP}(10 \%)^{1}$ & $-1,3$ & 5,9 & 14,6 & 23,2 & 31,5 & 39,8 & 47,7 & 55,3 & 62,5 & 69,7 \\
\hline $\operatorname{BLUP}(15 \%)^{1}$ & $-1,7$ & 5,4 & 13,9 & 22,4 & 30,7 & 38,7 & 46,3 & 53,7 & 60,9 & 67,7 \\
\hline \multirow[t]{2}{*}{$\operatorname{BLUP}(20 \%)^{1}$} & $-2,4$ & 5,0 & 13,0 & 21,3 & 29,4 & 37,6 & 45,5 & 53,2 & 60,5 & 67,4 \\
\hline & & \multicolumn{9}{|c|}{ Herdabilidade $($ Heritability $)=0,30$} \\
\hline $\operatorname{BLUP}(0 \%)$ & 1,9 & 12,0 & 23,9 & 35,6 & 47,0 & 57,5 & 67,6 & 77,5 & 86,5 & 95,3 \\
\hline $\operatorname{BLUP}(5 \%)$ & 1,5 & 11,6 & 23,6 & 34,9 & 45,8 & 56,1 & 66,5 & 76,1 & 85,4 & 94,1 \\
\hline $\operatorname{BLUP}(10 \%)$ & 1,0 & 11,0 & 22,6 & 33,9 & 44,4 & 55,1 & 65,4 & 75,1 & 84,7 & 93,6 \\
\hline $\operatorname{BLUP}(15 \%)$ & 0,6 & 10,6 & 21,9 & 32,7 & 43,4 & 53,7 & 63,7 & 73,4 & 82,5 & 91,1 \\
\hline \multirow[t]{2}{*}{$\operatorname{BLUP}(20 \%)$} & 0,3 & 10,2 & 21,4 & 32,1 & 42,5 & 52,7 & 62,6 & 72,0 & 80,7 & 89,3 \\
\hline & & \multicolumn{9}{|c|}{ Herdabilidade $($ Heritability $)=0,60$} \\
\hline $\operatorname{BLUP}(0 \%)$ & $-4,6$ & 8,9 & 23,2 & 37,1 & 50,7 & 63,9 & 76,2 & 88,1 & 99,1 & 109,8 \\
\hline $\operatorname{BLUP}(5 \%)$ & $-5,1$ & 8,4 & 22,4 & 36,1 & 49,3 & 62,0 & 74,3 & 86,2 & 97,5 & 108,1 \\
\hline $\operatorname{BLUP}(10 \%)$ & $-5,6$ & 7,6 & 21,2 & 34,9 & 47,9 & 60,3 & 72,0 & 83,5 & 94,5 & 105,2 \\
\hline BLUP(15\%) & $-6,2$ & 6,9 & 20,4 & 33,7 & 46,7 & 59,5 & 71,8 & 83,4 & 94,6 & 104,9 \\
\hline $\operatorname{BLUP}(20 \%)$ & $-6,7$ & 6,4 & 19,7 & 33,0 & 45,8 & 58,2 & 69,9 & 81,6 & 92,5 & 103,0 \\
\hline
\end{tabular}

${ }^{1}$ BLUP $=$ Melhor Predição Linear Não-Viesada com diferentes níveis de erros na seleção de machos.

${ }^{1}$ BLUP $=$ Best Linear Unbiased Prediction with different levels of errors in the selection of sires. 
CARNEIRO et al.

Tabela 2 - Eficiência do BLUP, com 0, 5, 10, 15 e 20\% de erro na seleção de machos, em relação ao BLUP com 0\% de erro Table 2 - Efficiency of BLUP with 0, 10, 15 and $20 \%$ of error in the selection of sires compared to BLUP with $0 \%$ of error

Método de seleção

Selection method

Generation

\begin{tabular}{|c|c|c|c|c|c|c|c|c|c|c|}
\hline & 1 & 2 & 3 & 4 & 5 & 6 & 7 & 8 & 9 & 10 \\
\hline \multicolumn{11}{|c|}{ Herdabilidade $($ Heritability $)=0,10$} \\
\hline $\operatorname{BLUP}(0 \%)^{1}$ & 1,0 & 1,0 & 1,0 & 1,0 & 1,0 & 1,0 & 1,0 & 1,0 & 1,0 & 1,0 \\
\hline $\operatorname{BLUP}(5 \%)^{1}$ & 0,96 & 0,97 & 0,96 & 0,97 & 0,98 & 0,98 & 0,98 & 0,98 & 0,99 & 1,0 \\
\hline $\operatorname{BLUP}(10 \%)^{1}$ & 0,92 & 0,96 & 0,95 & 0,95 & 0,96 & 0,96 & 0,97 & 0,96 & 0,96 & 0,97 \\
\hline $\operatorname{BLUP}(15 \%)^{1}$ & 0,88 & 0,93 & 0,93 & 0,93 & 0,94 & 0,94 & 0,94 & 0,94 & 0,94 & 0,95 \\
\hline $\operatorname{BLUP}(20 \%)^{1}$ & 0,83 & 0,91 & 0,89 & 0,90 & 0,91 & 0,92 & 0,93 & 0,93 & 0,94 & 0,94 \\
\hline \multicolumn{11}{|c|}{ Herdabilidade $($ Heritability $)=0,30$} \\
\hline $\operatorname{BLUP}(0 \%)$ & 1,0 & 1,0 & 1,0 & 1,0 & 1,0 & 1,0 & 1,0 & 1,0 & 1,0 & 1,0 \\
\hline $\operatorname{BLUP}(5 \%)$ & 0,96 & 0,98 & 0,98 & 0,98 & 0,97 & 0,97 & 0,98 & 0,98 & 0,98 & 0,98 \\
\hline $\operatorname{BLUP}(10 \%)$ & 0,93 & 0,95 & 0,96 & 0,96 & 0,95 & 0,96 & 0,97 & 0,97 & 0,98 & 0,98 \\
\hline $\operatorname{BLUP}(15 \%)$ & 0,89 & 0,93 & 0,94 & 0,93 & 0,93 & 0,94 & 0,95 & 0,95 & 0,95 & 0,96 \\
\hline $\operatorname{BLUP}(20 \%)$ & 0,87 & 0,92 & 0,92 & 0,92 & 0,92 & 0,92 & 0,93 & 0,93 & 0,94 & 0,94 \\
\hline \multicolumn{11}{|c|}{ Herdabilidade $($ Heritability $)=0,60$} \\
\hline $\operatorname{BLUP}(0 \%)$ & 1,0 & 1,0 & 1,0 & 1,0 & 1,0 & 1,0 & 1,0 & 1,0 & 1,0 & 1,0 \\
\hline BLUP(5\%) & 0,93 & 0,97 & 0,97 & 0,97 & 0,97 & 0,97 & 0,97 & 0,98 & 0,98 & 0,98 \\
\hline BLUP $(10 \%)$ & 0,87 & 0,93 & 0,94 & 0,95 & 0,95 & 0,95 & 0,95 & 0,95 & 0,95 & 0,96 \\
\hline $\operatorname{BLUP}(15 \%)$ & 0,79 & 0,90 & 0,92 & 0,93 & 0,93 & 0,94 & 0,95 & 0,95 & 0,95 & 0,95 \\
\hline BLUP $(20 \%)$ & 0,73 & 0,88 & 0,90 & 0,91 & 0,92 & 0,92 & 0,92 & 0,93 & 0,94 & 0,94 \\
\hline
\end{tabular}

${ }^{1}$ BLUP $=$ Melhor Predição Linear Não-Viesada com diferentes níveis de erros na seleção de machos.

${ }^{1}$ BLUP = Best Linear Unbiased Prediction with different levels of errors in the selection of sires.

Tabela 3 - Redução média em ganho genético por geração (\%) do BLUP com 0, 5, 10, 15 e $20 \%$ de erro na seleção de machos, em relação ao BLUP com $0 \%$ de erro

Table 3 - Average reduction in the genetic gain by generation (\%) of BLUP, with 0, 10,15 and $20 \%$ of error in the selection of sires in relation to BLUP with $0 \%$ of error

Método de seleção Herdabilidade

Selectionmethod

\begin{tabular}{lccc}
\cline { 2 - 4 } & 0,10 & 0,30 & 0,60 \\
\hline BLUP $(0 \%)^{1}$ & 0,0 & 0,0 & 0,0 \\
BLUP $^{1}(5 \%)^{1}$ & 1,9 & 1,7 & 2,5 \\
BLUP $^{1}(10 \%)^{1}$ & 3,8 & 3,6 & 5,6 \\
BLUP $^{1}(15 \%)^{1}$ & 6,3 & 5,8 & 7,5 \\
${\text { BLUP }(20 \%)^{1}}^{1}$ & 8,4 & 7,5 & 9,8
\end{tabular}

${ }^{1}$ BLUP $=$ Melhor Predição Linear Não-Viesada com diferentes níveis de erros na seleção de machos.

${ }^{1}$ BLUP $=$ Best Linear Unbiased Prediction with different levels of errors in the selection of sires.

Os resultados observados mostram quedas nos ganhos fenotípicos atribuídos aos erros na seleção de machos ao longo das gerações estudadas. A redução no progresso genético, mesmo que pequena, quando se trata de características de importância econômica, é problema grave. FALCONER (1981) ressalta que, quando o resultado da seleção é questão de ganho econômico, mesmo pequeno incremento na resposta à seleção será de grande importância.

Outro fator importante a mencionar com rela- ção a este tipo de erro é que, apesar dos reprodutores, neste estudo, terem sido utilizados pouco intensivamente (1 macho : 10 fêmeas), deixando relativamente poucos descendentes ( 80 descendentes), os ganhos genéticos foram bastante reduzidos. Se forem considerados touros que participam de programas de inseminação artificial, os quais são utilizados em grande número de vacas, deixando muitos filhos em vários rebanhos, o impacto deste tipo de erro poderia ser bem maior.

\section{Conclusões}

Quanto maiores os níveis de erros na seleção dos machos, maiores foram as reduções nos ganhos genéticos, independente das herdablidades estudadas.

São necessárias futuras pesquisas que avaliem o efeito do erro na seleção de machos e fêmeas sobre os ganhos genéticos em sistemas nos quais os reprodutores são usados em maior intensidade (relação macho/fêmea).

\section{Referências Bibliográficas}

CARNEIRO, P. L. S. Efeito de erros de pedigree na avaliação genética animal. Viçosa, MG: Universidade Federal de Viçosa, 1998. 79 p. Dissertação (Mestrado em Genética e Melhoramento) - Universidade Federal de Viçosa, 1998.

EUCLYDES, R.F. Uso do sistema para simulação Genesys na avaliação de métodos de seleção clássicos e associados a marcadores moleculares. Viçosa, MG: Universidade Federal de Viçosa, 1996. 149 p. Tese (Doutorado em Genética e 
268 Rev. bras. zootec.

Melhoramento) - Universidade Federal de Viçosa, 1996.

FALCONER, D.S. 1981. Introdução à genética quantitativa. Tradução de M. A. Silva e J. C. Silva. Viçosa, MG: UFV, Impr. Univ., 279 p.

HENDERSON, C.R. Selection index and expected genetic advance. In: NATIONAL ACADEMY OF SCIENCE/ NATIONAL RESEARCH COUNCIL - NAS / NRC. Statistical genetics and plant breeding. - Washington, DC: p. 141-163, 1963.

HENDERSON, C.R. Sire evaluation and genetic trends. In: ANIMAL BREEDING GENETICS SYMPOSIUM IN HONOR OF Dr. J. L. LUSH, 1973, Blacksburg. Proceedings... Champaign: ASAS/ADSA, p. 10-41, 1973.

HENDERSON, C.R. 1974. General flexibility of linear model techniques for sire evaluation. J. Dairy Sci., 57:963-972.

HENDERSON, C.R. Avaliação de vacas e touros. In: SIMPÓSIO BRASILEIRO DE MELHORAMENTO GENÉTICO DE BOVINO LEITEIRO NOS TRÓPICOS, 1, 1983, Coronel Pacheco, MG. Anais... Coronel Pacheco, MG: EMBRAPA/ CNPGL, P.137-189, 1983.

LIMA, R.R. Influência de erros nos valores fenotípicos sobre estimativas de parâmetros e avaliação genética animal. Viçosa, MG: Universidade Federal de Viçosa, 1998. 87p. Dissertação (Mestrado em Genética e Melhoramento) Universidade Federal de Viçosa, 1998.
LONG, T.E., JOHNSON, R.K., KEELE, J.W. 1990. Effects of erros in pedigree on three methods of estimating breeding value for litter size, backfat and average daily gain in swine. J. Anim. Sci., 68:4069-4078.

MARTINS, E.N., LOPES, P.S., SILVA, M.A. et al. 1997. Uso de modelos mistos na avaliação genética animal. Cadernos Didáticos, Viçosa: UFV, n.18, $121 \mathrm{p}$.

VAN VLECK, L.D. 1970a. Misidentification in estimating the paternal sib correlation. J. Dairy Sci., 53:1469-1474.

VAN VLECK, L.D. 1970b. Misidentification and sire evaluation. J. Dairy Sci., 53:1697-1702.

Recebido em: 18/06/98

Aceito em: 26/10/98 